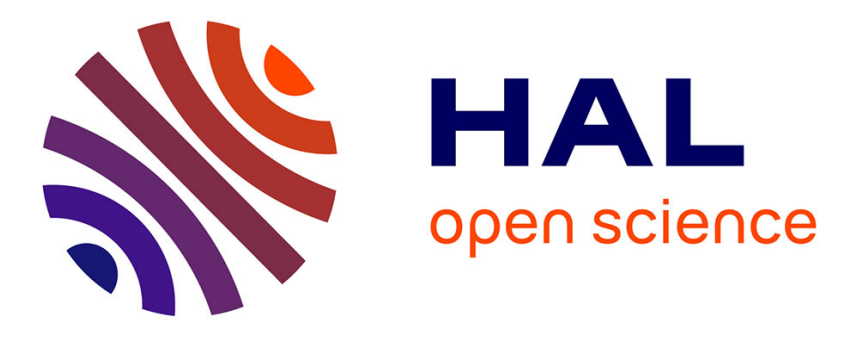

\title{
On contact transition for nonholonomic mobile manipulators
}

Vincent Padois, Jean-Yves Fourquet, Pascale Chiron, Marc Renaud

\section{To cite this version:}

Vincent Padois, Jean-Yves Fourquet, Pascale Chiron, Marc Renaud. On contact transition for nonholonomic mobile manipulators. Ang, M.H. and Khatib, O. The 9th International Symposium on Experimental Robotics (ISER 2004), Springer, pp.207 - 216, 2006, Springer Tracts in Advanced Robotics, Volume 21, 10.1007/11552246_20 . hal-00624296

\section{HAL Id: hal-00624296 https://hal.science/hal-00624296}

Submitted on 16 Sep 2011

HAL is a multi-disciplinary open access archive for the deposit and dissemination of scientific research documents, whether they are published or not. The documents may come from teaching and research institutions in France or abroad, or from public or private research centers.
L'archive ouverte pluridisciplinaire HAL, est destinée au dépôt et à la diffusion de documents scientifiques de niveau recherche, publiés ou non, émanant des établissements d'enseignement et de recherche français ou étrangers, des laboratoires publics ou privés. 


\title{
On contact transition for nonholonomic mobile manipulators
}

\author{
V. Padois ${ }^{1}$, J-Y. Fourquet ${ }^{1}$, P. Chiron ${ }^{1}$, and M. Renaud ${ }^{2}$ \\ 1 LGP-ENIT,Tarbes, France \\ \{vpadois,fourquet,pchiron\}@enit.fr \\ 2 LAAS-CNRS,Toulouse, France \\ renaud@laas.fr
}

\begin{abstract}
This work is devoted to planning and execution of complex missions in Robotics. Robotics has evolved from an industrial, repetitive framework to application domains with much more variability of tasks, with increasing complexity in uncertain environment. This is clearly the case for Service Robotics e.g. but even industrial robots have now to work in environment not totally calibrated for the task they have to perform. The result is that the classical decomposition in static ordered local tasks cannot apply in presence of such a variability. It has a poor dynamic performance and cannot cope with uncertainties. Our work is organized around a complex mission: "Go to the blackboard and write" for mobile manipulators that have capabilities of locomotion and manipulation. It is a simple and intuitive example of a complex mission that relies on different sensors, exhibits different operating modes and needs to switch between different feedbacks and set-points. Our approach is based on Hybrid Dynamical Systems. It is focused on dynamical sequencing of control laws that ensures good transients, robustness and allows to update the mission at every transition from one mode to another. Simulation have been realized with matlab Simulink and Stateflow toolboxes and experimental validation is developed within the $G^{e n}$ om controller on the $\mathrm{h}_{2}$ bis nonholonomic mobile manipulator.
\end{abstract}

\section{Introduction}

For many years, research community in robotics has proposed numerous solutions allowing manipulators and, more recently, mobile manipulators to solve specific tasks. In the literature, contributions concerning the motion in free space of the endeffector, whose location is denoted $\boldsymbol{\xi}$, are clearly separated from the ones dealing with constrained motion for which contact force $\mathbf{f}$ is imposed together with $\boldsymbol{\xi}$. In the first ones, it is assumed that no contact force can occur whereas the second category considers that the contact is realized from the beginning. Regarding manipulators, many modeling issues - at dynamic and kinematic level - and related control schemes can be found in reference textbooks $[15,14,9]$. Methods and models have also been proposed to deal with the control of mobile manipulators. Modeling issues can be found in $[2,4]$ and control issues are exposed in [16,18].

Thus, the approach generally adopted when considering a complex problem is to decompose it in a sequence of elementary subproblems, and then to solve independently each subproblem. This approach has the merit to permit the study of each 
step of the sequence in a simple way since it disregards the other steps. It is particularly suited when the environment can be adapted to the task and when this task is repetitive enough to deserve ad hoc tuning. These features are usual in an industrial framework that requires a low versatility of the robotic system.

Now, new directions, both in service and manufacturing robotics, lead to embed these techniques in missions where uncertainty and variability are much more important. Wheeled locomotion, for instance, naturally produces positioning uncertainty. Environment is less calibrated and tasks are less repetitive. At the same time, it seems pertinent to build the solution from the library of classical methods by chaining them dynamically. So, it is necessary to adapt and combine existing strategies for local tasks in order to realize complex missions with good transients.

Even if the objectives of our work are more general, the work presented here is sufficiently rich to point out problems and features of a more generic complex mission in robotics. It is organized around one kind of mission:

From an imposed end-effector location, a free space motion is followed by a trajectory tracking over a surface along which a normal force is imposed,

and around one kind of system: A nonholonomic mobile manipulator, kinematically redundant, built from a serial arm mounted on a wheeled mobile platform. This system is equipped with a force sensor that measures contact forces at the end-effector.

We made the choice of a description based on the Hybrid Dynamical Systems formalism where switchings among a finite number of modes occur when particular events are detected. Modes are linked to different set-points and control laws. The emphasis is made on the dynamical nature of the sequence of elementary modes and more particularly on the contact transition problem (i.e. transition from free space to constrained effector motion). This issue has been emphasized very early for manipulators [12] but only a little number of authors has actually studied it (e. $g$. $[10,7,17])$. These works mainly deal with stability issues and impact modeling. Recently, Yu and Pagilla [20] have presented a method that allows to cope with geometric uncertainties of the contact surface location. Doh et al. [5] have also proposed a specific control law that aims at avoiding large bounces during hard contact transitions. With regard to mobile manipulators, the contact transition problem is an emerging field of research that has not been extensively studied yet. Nonetheless, one can refer to the work of Nagatani et al. [11] focused on static chaining of primitives ("Open the door") but also to the work of Kang et al. [8] focused on the utilization of inertial effect when controlling holonomic mobile manipulators.

\section{Structuring and planning the mission}

In our approach a mission is described by $k$ modes or local tasks, and by a switching pattern that describes the sequencing of the local tasks. The switching pattern can be represented using a finite state machine. States corresponds to modes and transitions are activated by events: time elapsed or detection of a sensor threshold value. Using this formal description and the mission parameters, a first off-line planning step 
leads to "a perfect plan" that describes the ideal temporal evolution of the variables to control (i.e. the set-points or trajectories to track) and contains the calculated threshold values and time windows needed to define the transition conditions between modes. A state machine is then instantiated with these values. During the mission, this instantiated state machine creates indicators used to switch between the different phases of the plan and among a set of control schemes. At each transition, the "perfect plan" is updated thanks to the different signals and values of the variables describing the mission $(e . g$. the relative location of the end-effector in the environment frame is updated at contact time).

This update allows to cope with uncertainties due to poor parameter estimations of both the robot (drift of the platform, sensors resolution) and the environment. Thus, transition from a task to the following one occurs asynchronously but in a predictable time (or space) interval. Any large drift in the transition date or in the value of a controlled variable corresponds to an anomaly that cannot be interpreted as the result of these uncertainties and leads to a premature stop of the mission.

\section{Modeling of a mobile manipulator}
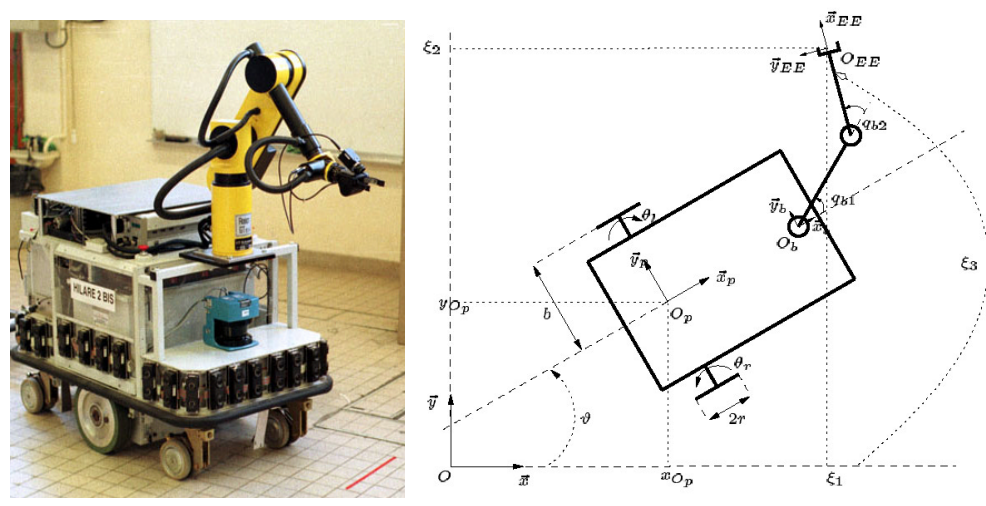

Figure1. (left) $\mathrm{H}_{2}$ bis: a 3-dimensional mobile manipulator; (right) A planar mobile manipulator.

We consider the case of mobile manipulators composed of a mobile platform with two independent driving wheels and a serial manipulator with $n_{b}$ joints. Such a system is depicted on figure 1 in a planar (right) or in a 3-dimensional version (left). This latter is the one used for the experiments. It has velocity controlled joints with high ratio gear reduction (i. e. the torque at each joints cannot directly be controlled) and so needs to rely on kinematic control schemes. Thus, we hereafter present some kinematics modeling results regarding mobile manipulators based on Bayle et al. work [2,3]. Nonetheless, dynamics are taken into account in impact reduction control scheme.

The configuration of such a mobile manipulator is completely defined using vec-

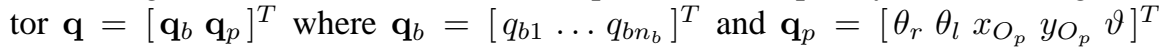


respectively represent the manipulator configuration and the platform configuration. Its end-effector location (i. e. location of $\mathcal{R}_{E E}=\left(O_{E E}, \vec{x}_{E E}, \vec{y}_{E E}, \vec{z}_{E E}\right)$ in $\mathcal{R}=(O, \vec{x}, \vec{y}, \vec{z}))$ can be described using a minimal set of parameters $\boldsymbol{\xi}=\left[\xi_{1} \ldots \xi_{m}\right]^{T} . \boldsymbol{\xi}$ is expressed as a non linear function of $\mathbf{q}$. Differentiating it, the relation between $\dot{\xi}$ and $\dot{\mathbf{q}}$ is given by:

$$
\dot{\boldsymbol{\xi}}=J(\mathbf{q}) \dot{\mathbf{q}} \text { with } J(\mathbf{q}) \text { a } m \times n \text { matrix and } n=n_{b}+5 .
$$

In addition, the rolling without slipping constraint acting on the platform's wheels is nonholonomic and so, components of $\dot{\mathbf{q}}$ cannot vary independently. Then, one can define a vector $\mathbf{u}=\left[\mathbf{u}_{b} \mathbf{u}_{p}\right]$ of independent parameters (i. e. taking the nonholonomic constraints into account) such as: $\dot{\mathbf{q}}=T(\mathbf{q}) \mathbf{u}$.

Defining $\bar{J}(\mathbf{q})=J(\mathbf{q}) T(\mathbf{q})$ equation (1) becomes: $\dot{\boldsymbol{\xi}}=\bar{J}(\mathbf{q}) \mathbf{u}$. This equation completely describes the mobile manipulator kinematics. This model is called instantaneous posture kinematic model in [1]. A natural choice for $\mathbf{u} \in \mathcal{R}^{\bar{n}}\left(\bar{n}=n_{b}+2\right)$ is $\mathbf{u}_{b}=\mathbf{q}_{b}$ and $\mathbf{u}_{p}$ can be chosen as $\left[\dot{\theta}_{r} \dot{\theta}_{l}\right]^{T}$ or $[v \omega]^{T}$ where $v$ and $\omega$ are respectively the linear and angular velocities of the platform.

When $m<\bar{n}$, the mobile manipulator is said to be kinematically redundant. This property provides the capability to choose a particular kinematic control vector $\mathbf{u}$ among those giving the prescribed end-effector velocity $\dot{\xi}$ by using the relation:

$$
\mathbf{u}=\bar{J}(\mathbf{q})^{\sharp} \dot{\boldsymbol{\xi}}+\left(I-\bar{J}(\mathbf{q})^{\sharp} \bar{J}(\mathbf{q})\right) \mathbf{z},
$$

where $\bar{J}(\mathbf{q})^{\sharp}$ is any generalized inverse of $\bar{J}(\mathbf{q})$ and $\mathbf{z}$ any $\bar{n} \times 1$ vector. Note that, among the generalized inverses, authors generally choose the pseudo-inverse [6] and that the second right-hand term of equation (2) gives rise to the so-called "internal motion" control term since it does not provide any end-effector velocity.

\section{Control schemes and use of the redundancy for the "Go to the blackboard and write" mission}

An ad hoc decomposition of this mission is given by the finite state machine on figure 2.

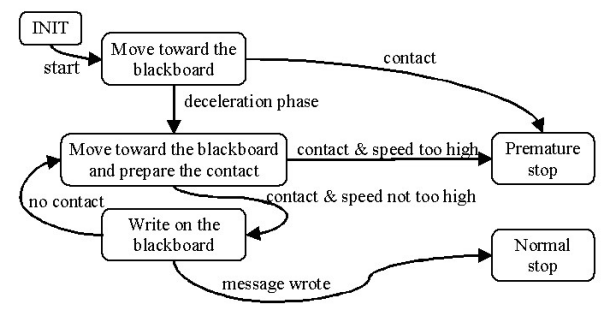

Figure2. High-level finite state machine for the "go to the blackboard and write mission" 


\subsection{Operational kinematic control}

Operational kinematic control uses a two stages control structure. The first stage is at the actuator level. Each actuator has its own velocity control loop. In our case, these controllers are digital PID regulators. The second stage is at end-effector level. Given $\dot{\xi}^{*}$ and $\xi^{*}$ desired end-effector speed and location to track, and a positive definite weighting matrix $W_{\text {reg }}$, the control vector defined by:

$$
\mathbf{u}=\bar{J}(\mathbf{q})^{\sharp}\left(\dot{\boldsymbol{\xi}}^{*}+W_{\text {reg }}\left(\boldsymbol{\xi}^{*}-\boldsymbol{\xi}\right)\right)+\left(I-\bar{J}(\mathbf{q})^{\sharp} \bar{J}(\mathbf{q})\right) \mathbf{z},
$$

ensures an asymptotic decreasing of $e=\boldsymbol{\xi}^{*}-\boldsymbol{\xi}$ toward 0 and $\mathbf{z}$ is chosen such as to minimize a scalar function (a potential function) $\mathcal{P}(\mathbf{q})$ [3].

\subsection{Hybrid speed / force controller}

The hybrid speed / force controller is a modified version of the well known work by Raibert and Craig [13].

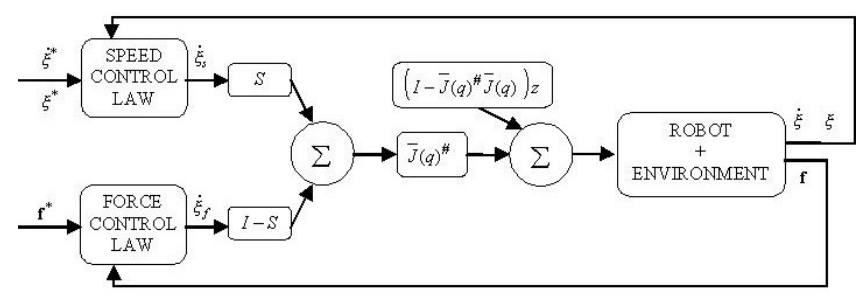

Figure3. Hybrid speed / force scheme control.

Once the contact established, the robot's end-effector cannot independently exert a displacement and a force in the same direction. One has to choose the direction to be position or speed controlled and the direction to be force controlled. $\vec{n}_{c}$ being the unit vector normal to the environment surface at contact point, a smart choice for the reference frame $\mathcal{R}$ is $\vec{x}=-\vec{n}_{c}$. Thus, one can create a $m \times m$ diagonal selection matrix $S$ where ones or zeros are placed on the diagonal respectively to indicate whether the component of $\boldsymbol{\xi}$ corresponding to the line in $S$ is speed or force controlled. The control vector is then calculated as the sum of three terms:

$$
\begin{aligned}
& \mathbf{u}=\mathbf{u}_{s}+\mathbf{u}_{f}+\mathbf{u}_{r} \text { with } \\
& \mathbf{u}_{s}=\bar{J}(\mathbf{q})^{\sharp} S \dot{\boldsymbol{\xi}}_{s}, \mathbf{u}_{f}=\bar{J}(\mathbf{q})^{\sharp}(I-S) \dot{\boldsymbol{\xi}}_{f} \text { and } \mathbf{u}_{r}=\left(I-\bar{J}(\mathbf{q})^{\sharp} \bar{J}(\mathbf{q})\right) \mathbf{z} .
\end{aligned}
$$

$\dot{\boldsymbol{\xi}}_{s}$ and $\dot{\boldsymbol{\xi}}_{f}$ are the control vectors whose simple versions are given by:

$$
\dot{\boldsymbol{\xi}}_{s}=\dot{\boldsymbol{\xi}}^{*}+W_{\text {reg }}\left(\boldsymbol{\xi}^{*}-\boldsymbol{\xi}\right) \text {, and } \dot{\boldsymbol{\xi}}_{f}=W_{\text {reg }_{f}}\left(\mathbf{f}^{*}-\mathbf{f}\right),
$$

where $W_{\text {regs }}$ and $W_{\text {reg }_{f}}$ are two positive definite weighting matrices. A block description of this control scheme is given by figure 3 . 


\subsection{Use of redundancy}

We expose here a set of functions to optimize using the internal motion. Many other functions may be used but these ones seem to be relevant according to the considered mission.

Manipulability maximization: The manipulability notion was first introduced for manipulators ( $c f$. [19] for a detailed presentation of this notion) but was also extended to mobile manipulators in [3]. The different manipulability measures are quantitative indicators representing the ease to instantly move the end-effector in any direction. Maximizing any of these indicators tends to avoid singular configuration of the system and thus to avoid high joints speed.

Impact force reduction: During the transition tasks, it is interesting to re-configure the mobile manipulator using internal motion so as to give it good inertial properties. Results concerning holonomic mobile manipulators are presented in [8]. The notion of impulse force $\hat{\mathcal{F}}$ is used in[17] and is defined as:

$$
\hat{\mathcal{F}}=\lim _{\Delta t \rightarrow 0} \int_{t}^{t+\Delta t} \mathbf{f}(s) d s,
$$

where $t$ is the impact time. Using the dynamic model of the system (i. e. the model establishing the relation between physical effects of motion, actuating torques and contact forces at end-effector level), the magnitude $\hat{F}$ of $\hat{\mathcal{F}}$ is expressed as:

$$
\hat{F}=\frac{-(1+e) \mathbf{v}_{\text {impact }}^{T} \mathbf{n}_{\mathbf{c}}}{\mathbf{n}_{\mathbf{c}}^{\mathbf{T}} C(\mathbf{q}) \mathbf{n}_{\mathbf{c}}} .
$$

$\mathbf{v}_{\text {impact }}^{T}$ is defined as the linear speed of the end-effector at the impact time, $e$ is the restitution coefficient (i.e. a physical characteristic of the contact) and $C(\mathbf{q})$ is an inertia-related matrix.

To minimize $\hat{F}$, one can:

- plan a low impact speed but uncertainties in the plan execution may lead to higher values than the planned one,

- maximize, using internal motion, $\mathbf{n}_{\mathbf{c}}^{\mathbf{T}} C(\mathbf{q}) \mathbf{n}_{\mathbf{c}}$ which is configuration dependent. It directly acts on the inertial properties of the manipulator.

Collision avoidance: Techniques to avoid obstacles have extensively been studied in the case of mobile robots. However, the problem to solve here is more complicated since the end-effector is constrained by the manipulation task. Hence, to avoid collision of the platform with the surface of contact, the potential function to maximize is chosen as the distance between the surface and point $O_{p}$.

\section{Experimental system and simulation results}

Experiments are being developed on the system depicted on left figure 1. In order to easily test different control structures and schemes, we also developed a simulator using Matlab and Simulink. We hereafter detail the experimental and simulation frameworks and we then present some results obtained using the simulator. 


\subsection{Experimental framework}

The mobile platform is actuated using two independent driving wheels (i. e. an HILARE type platform). The manipulator is a 6R serial arm called GT6A. The whole system, $H 2 b i s$, is controlled using on board calculators equipped with the real time operating system VxWorks and running on Motorola 68000 and Apple Power PC cards.

Control algorithms are implemented using $G^{\text {en }}$ om, a generator of software control modules developed, as well as the robot, in the RIA team of the LAAS laboratory. At actuator level, the control sampling time is $5 \mathrm{~ms}$ (the smaller one admitted by the control modules) whereas at operational level we chose it to be either 10 or $20 \mathrm{~ms}$. The 6 -axis GIROBO force / torque sensor bandwidth is $100 \mathrm{~Hz}$.

\subsection{Simulation framework}

Robot: The robot is modeled at kinematic and dynamic levels as a continuous system. Actuators (dc motors) are modeled as second order linear systems and we take into account load torques due to dynamic effects of motion or due to contact. We also take into account saturations of the actuators.

Sensors: Force sensor inaccuracy is modeled together with additive noise. Force signal is sampled with a period of $10 \mathrm{~ms}$ to reflect the effects of its limited bandwidth.

Contact: Contact between the end-effector and the environment is locally modeled either as a \{spring // damper $\}$ system (i. e. Kelvin-Voigt visco-elastic model) or as a spring system. When running simulations, we consider that the values of the parameters characterizing the contact are unknown or poorly estimated.

Controllers: Actuators' controllers are modeled as digital PID regulators whose sampling time is $5 \mathrm{~ms}$. The operational level controller is also implemented as a digital controller. We consider its sampling time as an off-line tunable parameter whose minimum value is $10 \mathrm{~ms}$.

\section{3 "Go to the blackboard and write": Simulation results}

System depicted on right figure 1 is chosen for simulation. $\boldsymbol{\xi}$ is chosen as the end-

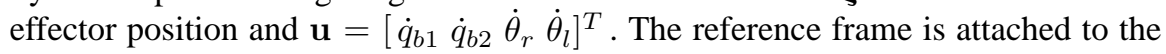
blackboard whose surface is defined by equation $x=0$. Thus, $\mathbf{f}$ is defined as the vector whose components are the normal and tangential force to the blackboard. Once the contact established, the normal direction is force controlled whereas the tangential direction is speed controlled.

Impact force reduction Simulation whose parameters are described in table 1 illustrates the effects of using kinematic redundancy to reduce impact peak force. 


\begin{tabular}{|c|c|c|c|c|c|}
\hline & real & \multicolumn{4}{|c|}{ estimated } \\
\hline contact point $[\mathrm{m}]$ & $\left.\begin{array}{ll}0 & 0\end{array}\right]^{T}$ & \multicolumn{4}{|c|}{$\left[\begin{array}{lll}0.05 & 0\end{array}\right]^{T}$} \\
\hline contact stiffness $k_{e}\left[N . m^{-1}\right]$ & 9000 & \multicolumn{4}{|c|}{$\hat{k}_{e}=k_{e}$} \\
\hline initial configuration $[\mathrm{rad}]$ and $[\mathrm{m}]$ & \multicolumn{5}{|c|}{$\left[\frac{\pi}{4} \frac{\pi}{4} 00-1.75-0.3 \frac{\pi}{3}\right]^{T}$} \\
\hline initial location $[m]$ & & \multicolumn{4}{|c|}{$-2 \quad 0.6]^{T}$} \\
\hline planned final location $[m]$ & & \multicolumn{4}{|c|}{$\left[\begin{array}{ll}0 & 0.5\end{array}\right]$} \\
\hline force set-point $[N]$ & & \multicolumn{4}{|c|}{$\left[\begin{array}{ll}10 & 0\end{array}\right]$} \\
\hline$W_{\text {reg }_{s}}\left[s^{-1}\right], W_{\text {reg }_{f}}\left[m \cdot N^{-1} \cdot s^{-1}\right], S$ & & ' & $\begin{array}{cc}\frac{10}{\hat{k}_{e}} & 0 \\
0 & 0\end{array}$ & , & $\begin{array}{ll}1 & 0 \\
0 & 0\end{array}$ \\
\hline Operational controller sampling time $[s]$ & \multicolumn{5}{|c|}{$20 \times 10^{-3}$} \\
\hline
\end{tabular}

Table1. Simulation parameters.
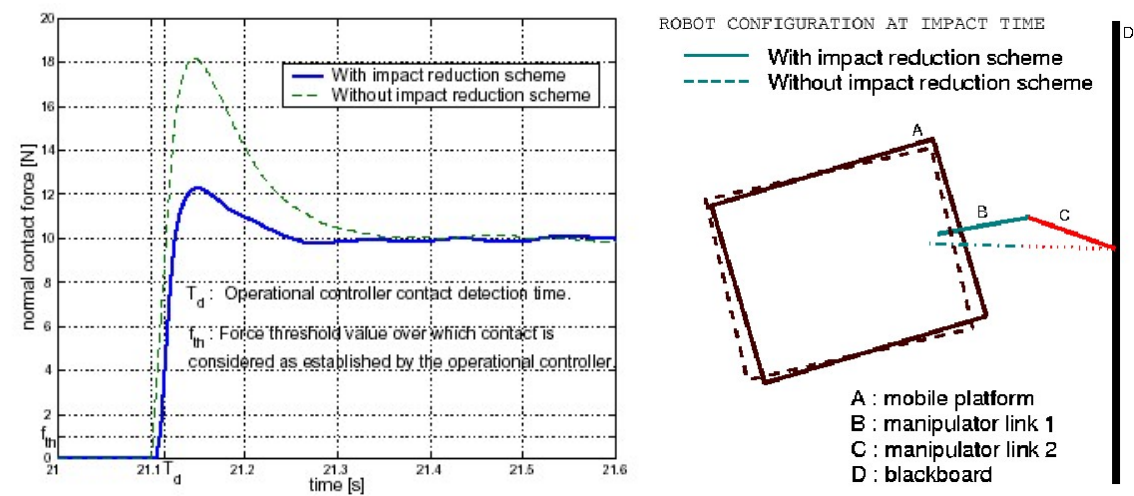

Figure4. Impact force reduction: (left) Normal contact force comparison. (right) Robot configuration at impact time.

Figures 4 and 5 have been obtained with two simulations: the first one is such that $\mathbf{z}=0$ in equation (3) whereas the second one uses a potential function aiming at minimizing impact forces ( $c f$. equation (8)). This potential is activated during a given period of time $\Delta T_{p}$ around the planned impact time.

Figure 4 (left) shows that force overshoot is decreased from $80 \%$ to $20 \%$ of the final desired value. Figures 4 (right) and 5 show how internal motions generated by the potential modify the evolution of each coordinate. Inertial characteristics depend on the arm configuration and it is expected that the use of this particular potential mainly affects the manipulator generalized coordinates. Indeed, $q_{b 1}$ and $q_{b 2}$ are the more influenced by the internal motion resulting from the chosen potential. However, the other coordinates are also modified since motion of the platform must compensate for manipulator motion.

According to the current configuration, the action of a potential may cause rough variations of generalized velocities that could be inconsistent with the velocity bounds of the system. Hence, the potential is introduced here with a time-varying coefficient ensuring velocity continuity and allowable velocities and accelerations for the system. 


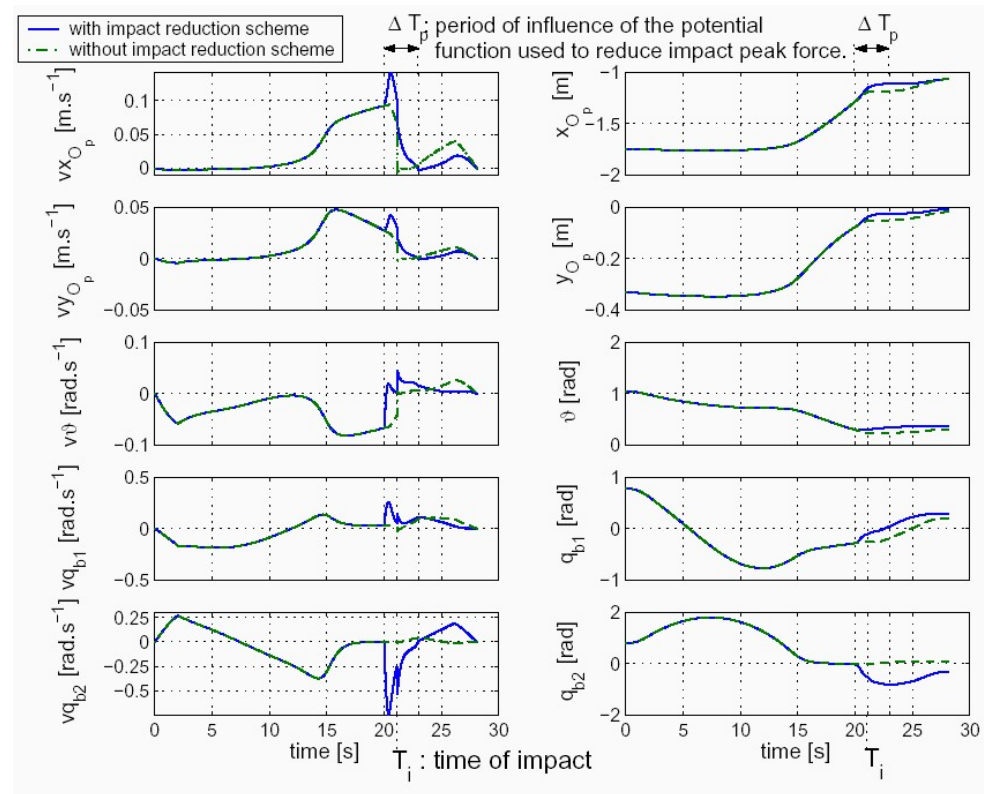

Figure5. Impact force reduction: Effects on the position and speed of the different robot's bodies.

\section{Conclusion}

The aim of this study was to propose a method to control dynamic contact transition for nonholonomic mobile manipulators. The method is based on the decomposition of complex mission in elementary tasks to sequence. A particular attention is paid to transition phases. A simulator, taking into account the physical system's constraints, was developed and allows to test different control structures and schemes. The obtained results show the efficiency of the proposed method. In particular, a smart use of the kinematic redundancy of the system allows to correctly control the contact transition phase. The principle of the proposed approach was validated using simulation and is being confirmed using experiments currently developed on robot $\mathrm{H}_{2}$ bis. Further developments are also led to propose a systematic method to generalize the sequence and scaling of different potential functions in a same mission.

Acknowledgment: This work is partially supported by the French CNRS Robea program within the project Dynamic sequencing of multi-sensor based tasks for complex motions execution in mobile robotics.

\section{References}

1. B. Bayle. Modélisation et commande cinématiques des manipulateurs mobiles à roues. PhD thesis, LAAS-CNRS, Toulouse, France, 2001. 
2. B. Bayle, J-Y. Fourquet, and M. Renaud. Kinematic modelling of wheeled mobile manipulators. In IEEE International Conference on Robotics and Automation, Taipei, Taiwan, September 2003.

3. B. Bayle, J-Y. Fourquet, and M. Renaud. Manipulability of wheeled mobile manipulation: application to motion generation. The International Journal of Robotics Research, vol. 22(7-8):565-581, July 2003.

4. G. Campion, G. Bastin, and B. D'Andréa Novel. Structural Properties and Classification of Kinematic and Dynamic Models of Wheeled Mobile Robots. IEEE Transactions on Automatic Control, 12:47-62, February 1996.

5. N. Doh, W.K. Chung, and Y. Youm. On hard contact force control. In IEEE/RSJ International Conference on Intelligent Robotic Systems, pages 1528-1533, Takamatsu, Japan, November 2000.

6. K. L. Doty, C. Melchiorri, and C. Boniveto. A theory of generalized inverses applied to Robotics. The International Journal of Robotics Research, 12(1):1-19, February 1993.

7. J. M. Hyde and M. R. Cutkosky. Controlling Contact Transition. IEEE Control Systems Magazine, 14(1):25-30, February 1994.

8. S. Kang, K. Komoriya, K. Yokoi, T. Koutoku, and K. Tanie. Utilization of inertial effect in damping-based posture control of mobile manipulator. In Proceedings of the 2001 IEEE International Conference on Robotics and Automation, pages 1277-1282, Seoul, Korea, 2001.

9. W. Khalil and E. Dombre. Modeling, Identification and Control of Robots. Hermes Penton Science, 2002.

10. J.K. Mills and D.M. Lokhorst. Control of robotic manipulators during general tasks execution: A discontinuous control approach. The International Journal of Robotics Research, vol. 12(2):146-163, April 1993.

11. K. Nagatani and S. Yuta. Door-opening behaviour of an autonomous mobile manipulator by sequence of action primitives. Journal of Robotic Systems, 13(11):709-721, 1996.

12. R.P. Paul. Problems and research issues associated with the hybrid control of force and displacement. In IEEE International Conference on Robotics and Automation, pages 1966-1971, Raleigh, North Carolina, March 1987.

13. M. H. Raibert and J. J. Craig. Hybrid position/force control of manipulators. ASME Journal of Dynamic Systems, Measurement and Control, 1981.

14. L. Sciavicco and B. Siciliano. Modelling and control of robot manipulators. Springer, 1999.

15. M. W. Spong and M. Vidyasagar. Robot Dynamics and Control. J. Wiley and Sons, 1989.

16. Y. Umeda, D. Nakamura, T. Mukarami, and K. Ohnishi. Hybrid position/force control of a mobile manipulator based on cooperative task sharing. In Proceedings of the 1999 IEEE International Symposium on Industrial Electronics, Bled, Slovenia, 1999.

17. I.D. Walker. Impact configurations and measures for kinematically redundant and multiple armed robot systems. IEEE Transactions on Robotics and Automation, vol. 12(5):670683, 1994.

18. Y. Yamamoto. Control and coordination of locomotion and manipulation of a wheeled mobile manipulator. PhD thesis, University of Pensylvania, 1994.

19. T. Yoshikawa. Foundations of robotics - Analysis and Control. The MIT Press, 1990.

20. B. Yu and P. R. Pagilla. A Switching Control Scheme for Constrained Robot Tasks. In Proceedings of the 2001 IEEE Conference on Control Applications, Mexico City, Mexico, September 2001. 